\title{
Cerebral Venous Sinus Thrombosis in Adults with Prothrombotic Conditions: A Systematic Review and a Case from Our Institution
}

Jack Komro ${ }^{1}$, Dawood Findakly ${ }^{2}$

1. Internal Medicine, Kirksville College of Osteopathic Medicine, A. T. Still University, Kirksville, USA 2. Internal Medicine, Creighton University Arizona Health Education Alliance/Valleywise Health Medical Center, Phoenix, USA

Corresponding author: Dawood Findakly, dawood_findakly@yahoo.com

\begin{abstract}
Cerebral venous sinus thrombosis (CVST) is a rare condition characterized by elevated intracranial pressure due to impaired cerebral venous drainage, potentially leading to life-threatening consequences. We searched the PubMed electronic database for 'cerebral venous sinus thrombosis' and 'prothrombotic' cases reported in adults (19+ years) and conducted a systematic review for the published literature in the English language pooled with a case from our institution. Data were analyzed regarding patient demographics, risk factors, clinical features, treatment modalities, and outcomes when available.
\end{abstract}

Thirty cases of CVST were identified (29 case reports, of whom two were described in a case series, and the one case from our institution). The patients' mean age was 39 years (range: 19 - 65). The male: female ratio was $1.14: 1$. The majority $(73.3 \%)$ had at least one preexisting risk factor, with prescription drug use being the most common risk factor (33.3\%) shared among all patients. Most patients (83.3\%) presented with at least two symptoms. The most common presenting symptoms were headache (70\%), gastrointestinal disturbance (50\%), and seizures (40\%). Focal deficits (36.7\%), vision disturbances (30\%), and altered consciousness (20\%) were the remaining presenting complaints. Twelve cases (40\%) commented on papilledema, with $10(83.3 \%)$ having papilledema present. Anticoagulation abnormalities were examined in 26 cases (86.7\%), out of which four cases (15.4\%) had isolated protein S (PS) deficiency, three cases (11.5\%) had isolated antithrombin III (ATIII) deficiency, and one case (3.8\%) had isolated protein C (PC) deficiency. The most common initial imaging modality ( 22 cases, $73.3 \%$ ), and most commonly used overall ( 23 cases, $76.7 \%$ ), was computed tomography (CT). Magnetic resonance imaging (MRI) was the second most common imaging modality for initial use (five cases, 16.7\%), diagnosis or confirmation of CVST (eight cases, 26.7\%), and overall (21 cases, 70\%). Heparin treatment was involved in the treatment of 18 cases (60\%), and warfarin treatment was used in 10 cases (33.3\%). Heparin-warfarin combination treatment was utilized in eight cases (26.7\%). Most patients survived ( 28 cases, $93.3 \%$ ), while the two remaining patients died secondary to brain death from the CVST (6.7\%).

The findings from this study highlight the clinical characteristics of CVST. Therefore, this study aims to increase awareness of this rare entity. Physicians should maintain a high index of suspicion in order to diagnose patients presenting in the proper clinical context, given this case shares various forms of presentations with other common clinical conditions but requires long-term anticoagulation.

Categories: Internal Medicine, Neurology, Hematology

Keywords: cerebral venous sinus thrombosis (cvst), antithrombin iii, protein c, protein s, papilledema, systematic review, hematology

\section{Introduction And Background}

Cerebral venous sinus thrombosis (CVST) is a relatively uncommon, but potentially life-threatening condition, that has variable and non-specific forms of clinical presentations [1-2]. Anticoagulants, mainly heparin agents, are used as first-line therapy, with most patients attaining an excellent response [3]. This study's objective is to review the patient characteristics, risk factors, clinical features, treatment modalities, and outcomes of CVST, a rare and life-threatening condition in patients with prothrombotic states.

\section{Review}

\section{Methods}

Search Strategy

The present study protocol adheres to the preferred reporting items for systematic reviews and metaanalyses (PRISMA) guidelines for reporting systematic review protocols. The PubMed database was searched for adults ( $\geqslant 19$ years old) and case reports in English using the terms 'cerebral sinus venous thrombosis' and 'prothrombotic' as keywords. Reference lists were also examined to identify relevant case reports. All fulltext published cases were selected, and the authors independently assessed cases for inclusion.

Data Extraction and Analysis

All studies evaluating CVST with prothrombotic abnormalities were screened, with the selection of only those reports containing data on demographic information, clinical features, prothrombotic laboratory results, and diagnostic imaging. Unrelated case reports and those without prothrombotic lab results were excluded (Figure 1). Data are expressed in descriptive statistics using central tendency and dispersion measures. 


\section{Cureus}

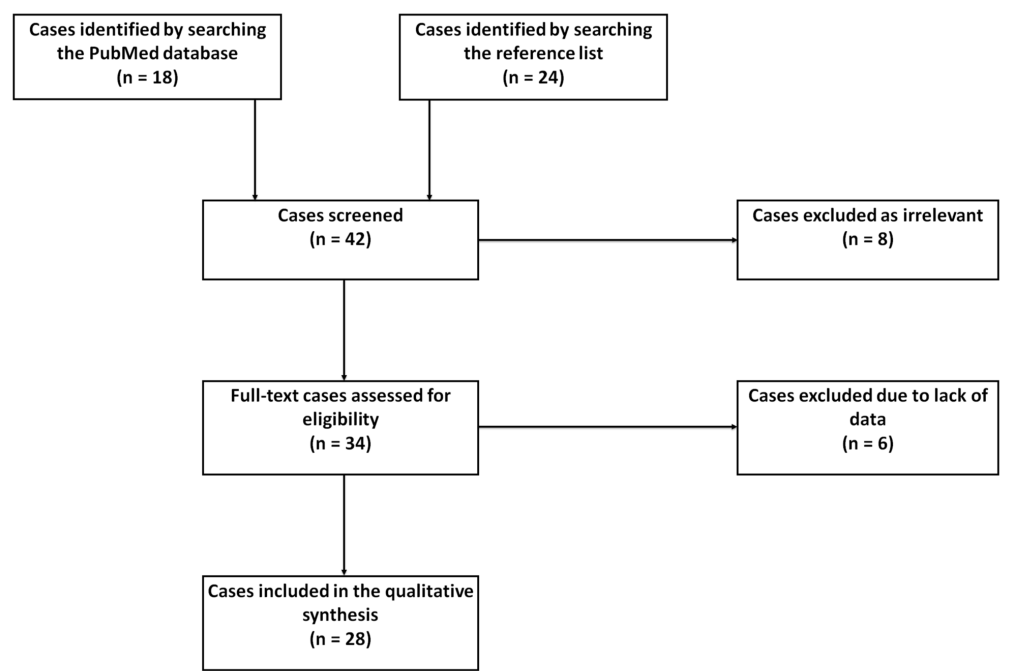

FIGURE 1: The PRISMA flow diagram for the systematic review detailing the association of CVST with prothrombotic abnormalities

CVST: cerebral venous sinus thrombosis; PRISMA: preferred reporting items for systematic reviews and meta-analyses

\section{Results}

A total of 42 case reports of CVST with prothrombotic laboratory results were screened, with 28 publications ultimately included [4-31]. One case series described two cases, both of which were included, yielding 29 cases for this systematic review. With the addition of a case from our institution, a total of 30 case reports were analyzed. The demographics, clinical features, and outcomes of the 30 cases are summarized in Table 1.

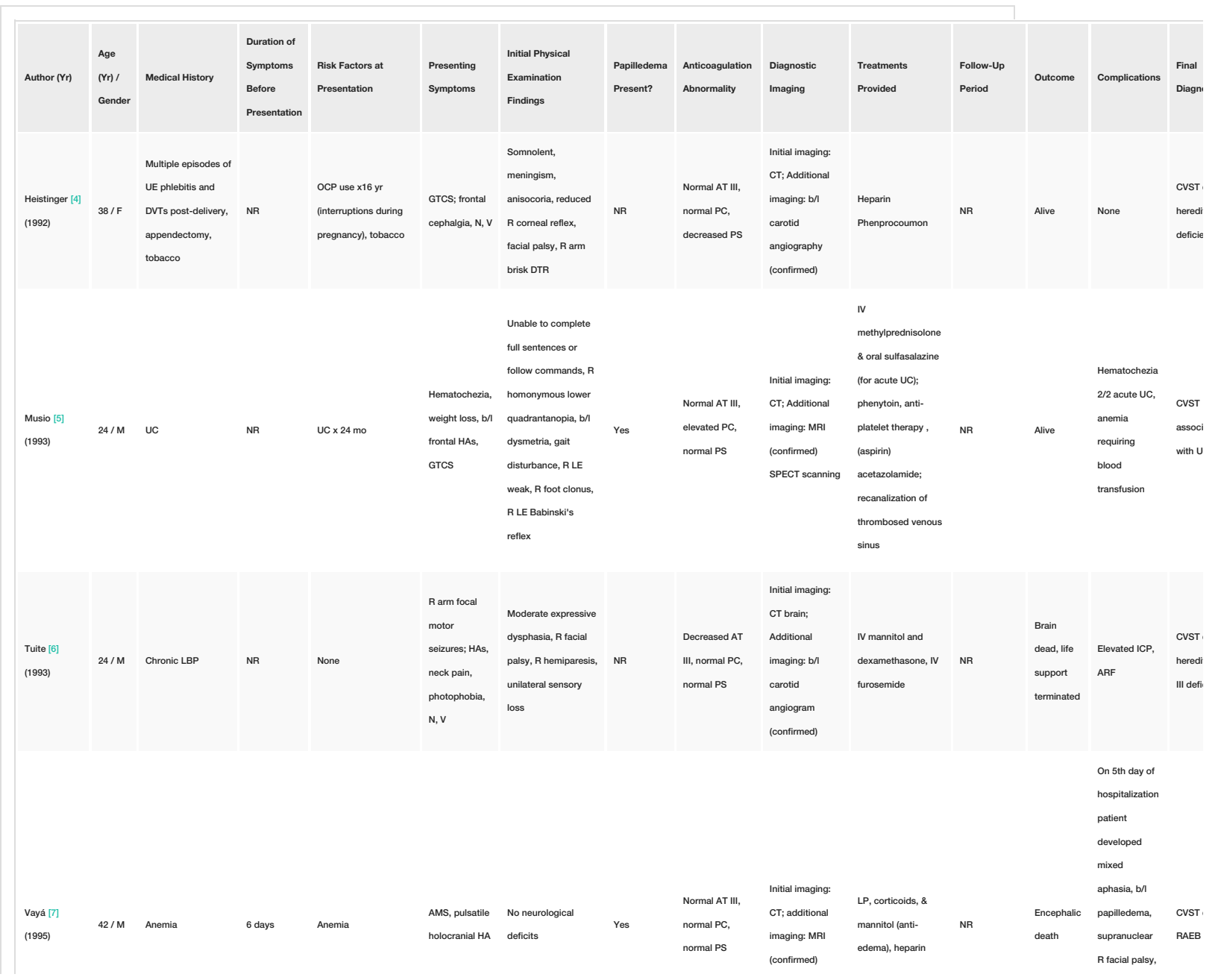




\section{Cureus}

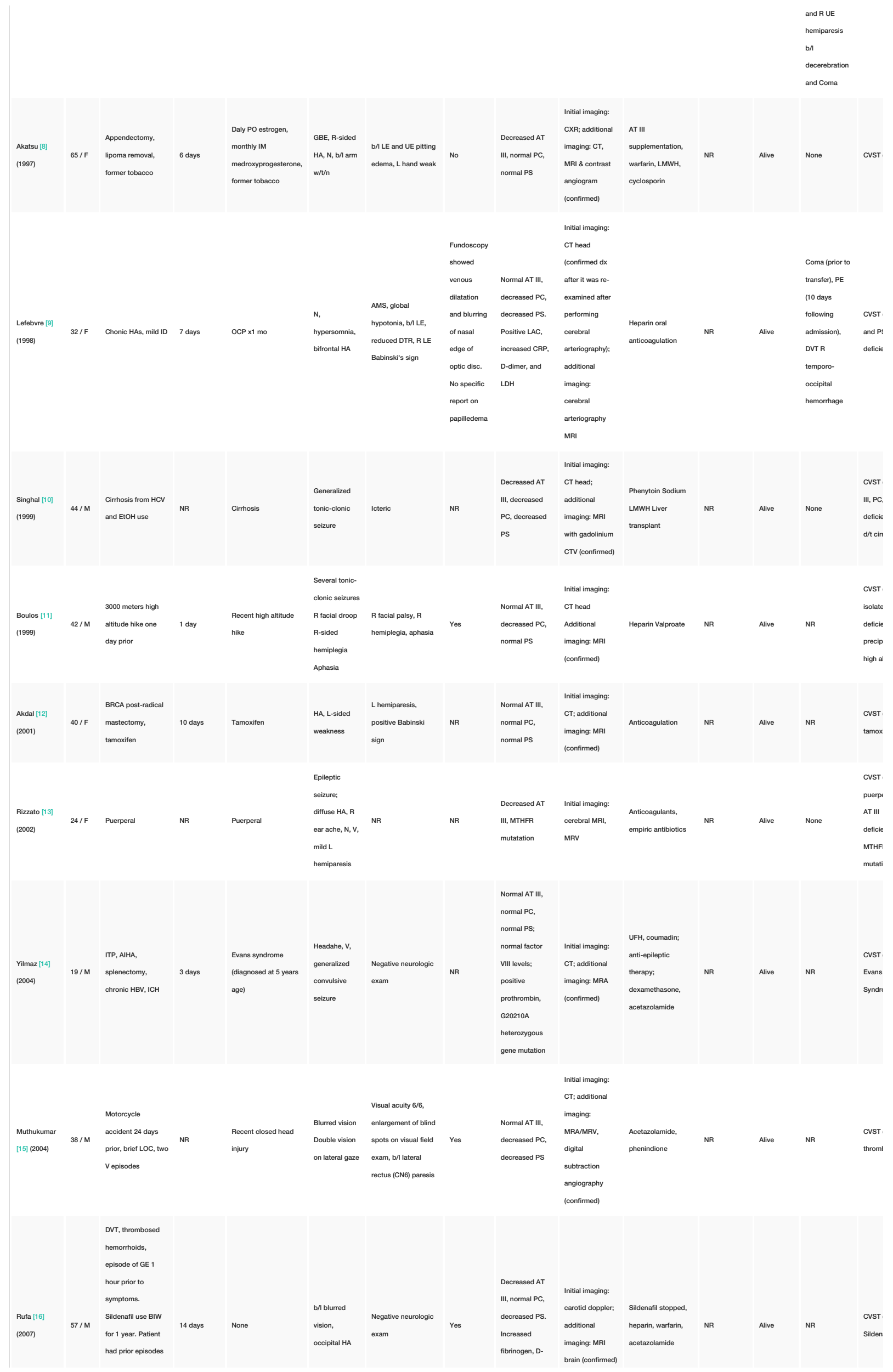




\section{Cureus}

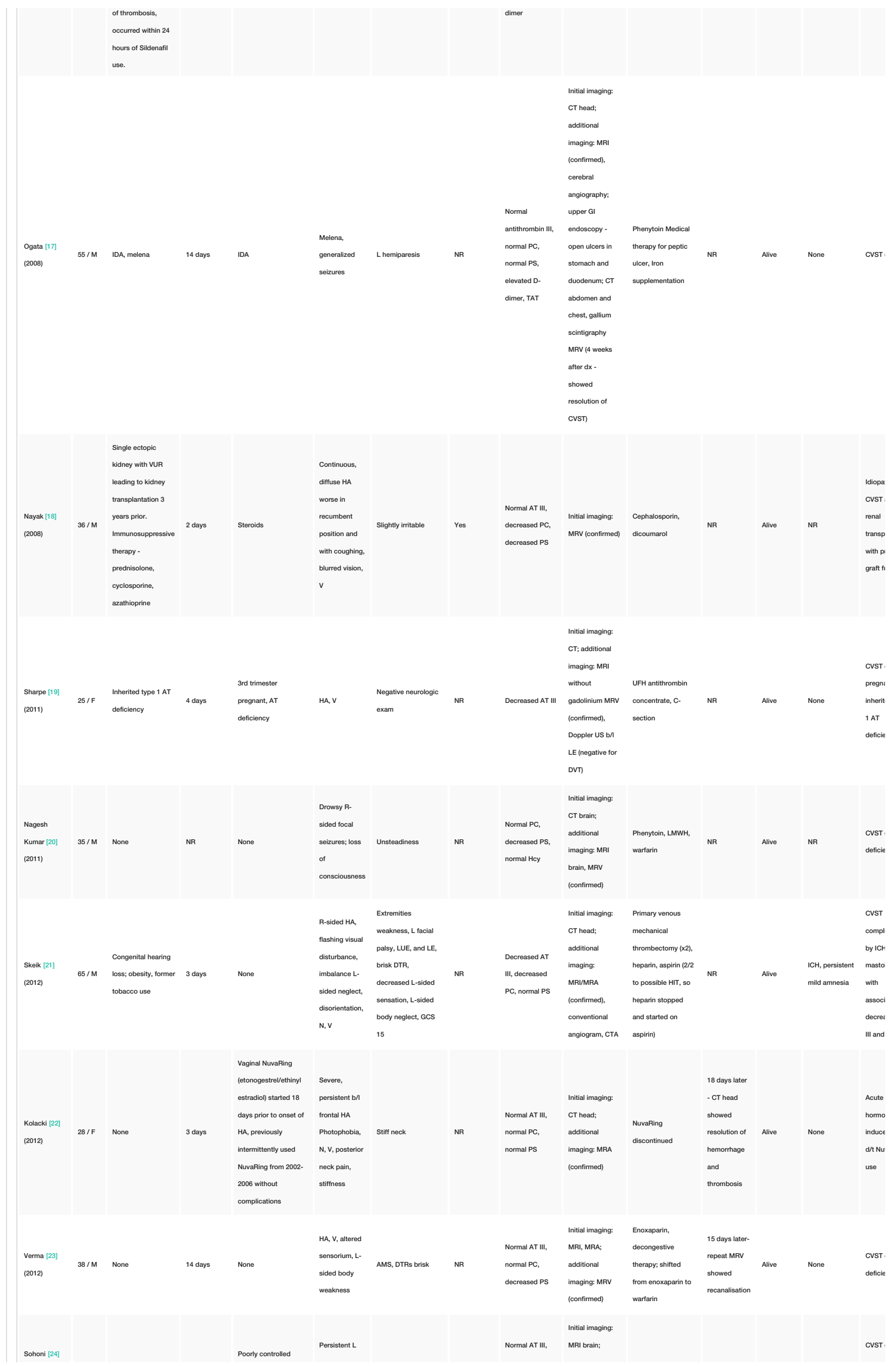




\section{Cureus}

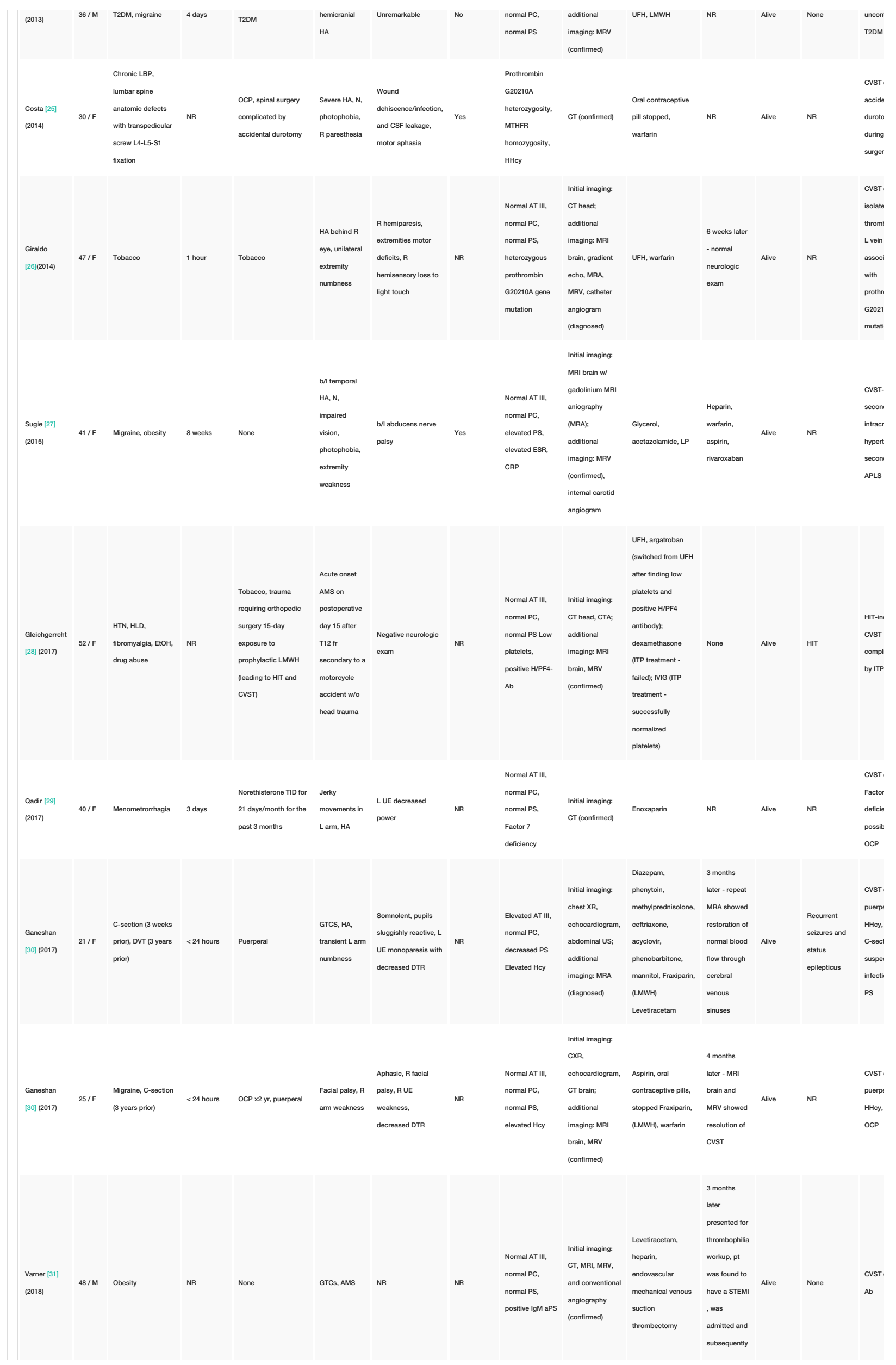




\section{Cureus}

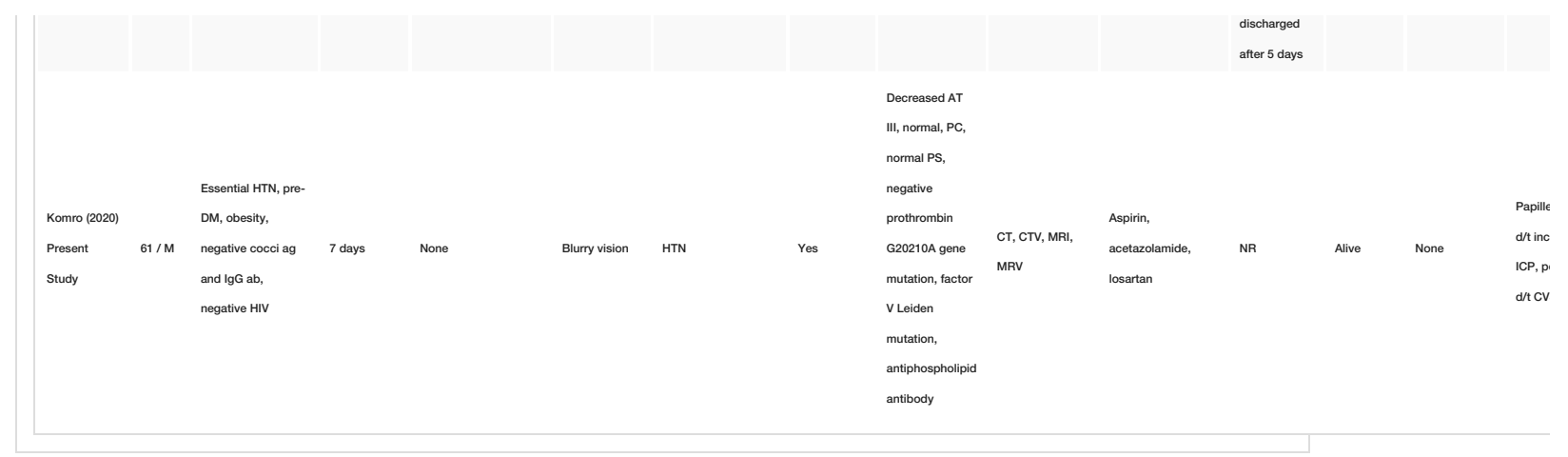

TABLE 1: Summary of the Clinical Characteristics, Risk Factors, Diagnostic, Management, and Outcomes of CVST Case Reports Included in the Systematic Review

AIHA: autoimmune hemolytic anemia; AMS: altered mental status; APLS: antiphospholipid syndrome; aPS: phosphatidylserine; aPS-Ab: antiphosphatidylserine antibodies; ARF: acute renal failure; AT: antithrombin; AT III: antithrombin III; BIW: two times weekly; b/l: bilateral; BRCA: breast cancer; CRP: C-reactive protein; cocci ag: coccidioides antigen; CT: computed tomography; CTA: computed tomography angiography; CVST: cerebral sinus venous thrombosis; DM: diabetes; d/t: due to; DTR: deep tendon reflex; DVT: deep venous thrombosis; EtOH: alcohol; F: female; fr: fracture; GBE: generalized body edema; GCS: Glasgow Coma Scale; GE: gastroenteritis; GTCS: generalized tonic-clonic seizure; HA: headache; HBV: hepatitis B virus; HCV: hepatitis C virus; Hcy: homocysteine; HHcy: hyperhomocysteinemia; HIT: heparin-induced thrombocytopenia; HIV: human immunodeficiency virus; HLD: hyperlipidemia; H/PF4-Ab: human platelet factor 4 antibody; HTN: hypertension; ICH: intracranial hemorrhage; ICP: intracranial pressure; ID: intellectual disability; IDA: iron deficiency anemia; IgG: immunoglobulin G; IgM: immunoglobulin M; ITP: immune thrombocytopenia; IVIG: intravenous immunoglobulin G; L: lumbar; LAC: lupus anticoagulant; LBP: low back pain; LDH: lactate dehydrogenase; LE: lower extremity; LMWH: low-molecular weight heparin; LOC: loss of consciousness; LP: lumbar puncture; LUE: left upper extremity; M: male; Mo: month; MRV: magnetic resonance venogram; MTHFR: methylene-tetrahydrofolate reductase; N: nausea; NR: not reported; NS: nephrotic syndrome; OCP: oral contraceptive use; PC: protein C; PE: pulmonary embolism; PS: protein S; R: right; RAEB: refractory reported; NS: nephrotic syndrome; OCP: oral contraceptive use; PC: protein C; PE: pulmonary embolism; PS: protein S; R: right; RAEB: refractory
anemia with excess of blasts; STEMI: ST-segment elevation myocardial infarction; TAT: thrombin-antithrombin III complex; T2DM: type 2 diabetes anemia with excess of blasts; STEMI: ST-segment elevation myocardial infarction; TAT: thrombin-antithrombin III complex; T2DM: type 2 dab
mellitus; TID: three times daily; UC: ulcerative colitis; UE: upper extremity; UFH: unfractionated heparin; US: ultrasound; V: vomiting; VUR: vesicoureteral reflux; w/o: without; Yr: year

The mean age at presentation was 39 years old (range: 19 - 65), with 24 (80\%) being less than 50 years old. There were 16 male ( $53.3 \%$ ) and 14 female (46.7\%) patients (Figure 2 ). The majority (73.3\%) had at least one preexisting risk factor (Figure 3). Prescription drugs were the most common risk factor (33.3\%) shared among all patients. A history of tobacco smoking was reported in four cases (13.3\%).

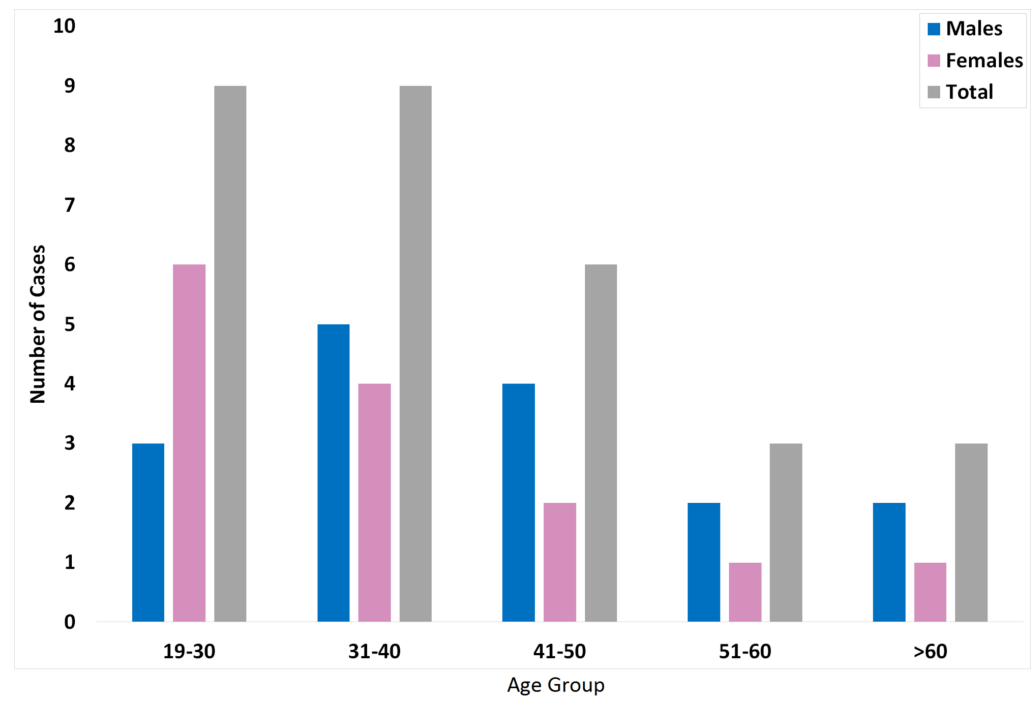

FIGURE 2: Age and gender distribution of adults with CVST. The bar graph represents the number of patients with CVST for the specific age and gender group 


\section{Cureus}

Prescription Drugs

Pregnancy/Puerperium

Tobacco Use

Mechanical Precipitants

Hematologic Condition

Inflammatory Diseases

Other Disorders
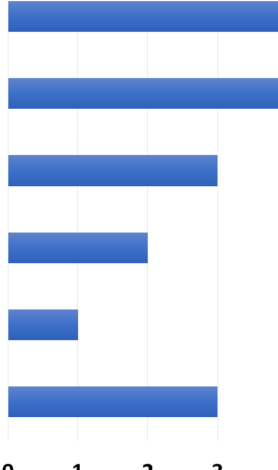

$\begin{array}{lllllll}4 & 5 & 6 & 7 & 8 & 9\end{array}$

FIGURE 3: Risk factor frequency in 30 patients with cerebral sinus venous thrombosis (CVST)

Among females, 11 (78.6\%) reported having gender-specific risk factors. Six (54.5\%) were receiving exogenous estrogen hormone therapy (EEHT), four (36.3\%) were pregnant or puerperal patients, and one (9.1\%) was receiving norethisterone therapy. Three case reports (10\%) involved mechanical precipitants. Other disorders, including congenital heart disease, thyroid disease, Evans syndrome, diabetes, and cirrhosis, were reported in three cases $(10 \%)$. The least common risk factors were a preexisting hematologic condition (two cases, $6.7 \%$ ) or inflammatory disease (one case, 3.3\%).

Of the 20 cases (66.7\%) that reported the duration of symptoms, most (55\%) had symptoms between two to seven days at presentation. Four patients (20\%) presented earlier with symptoms up to one day, while five patients (25\%) presented later with symptoms lasting at least eight days. One patient (5\%) had symptoms more than two weeks, not presenting until two months after symptom onset (Table 2).

\begin{tabular}{|l|l|}
\hline Duration & The fraction in each category $(\%)$ \\
\hline $0-1$ day & $4 / 20(20.0 \%)$ \\
\hline $2-7$ days & $11 / 20(55.0 \%)$ \\
$8-14$ days & $4 / 20(20.0 \%)$ \\
$>2$ weeks & $1 / 20(5.0 \%)$ \\
\hline
\end{tabular}

TABLE 2: The Duration of Symptoms Before the Patients' Presentation

Most patients (83.3\%) presented with at least two symptoms (Figure 4). The most common presenting symptoms were headache (70\%), gastrointestinal disturbance (50\%), and seizures (40\%). Focal deficits (36.7\%), vision disturbances (30\%), and altered consciousness / confusion / disorientation (20\%) were the remaining presenting complaints. Twelve cases (40\%) commented on papilledema, with 10 patients (83.3\%) having papilledema present. 


\section{Cureus}

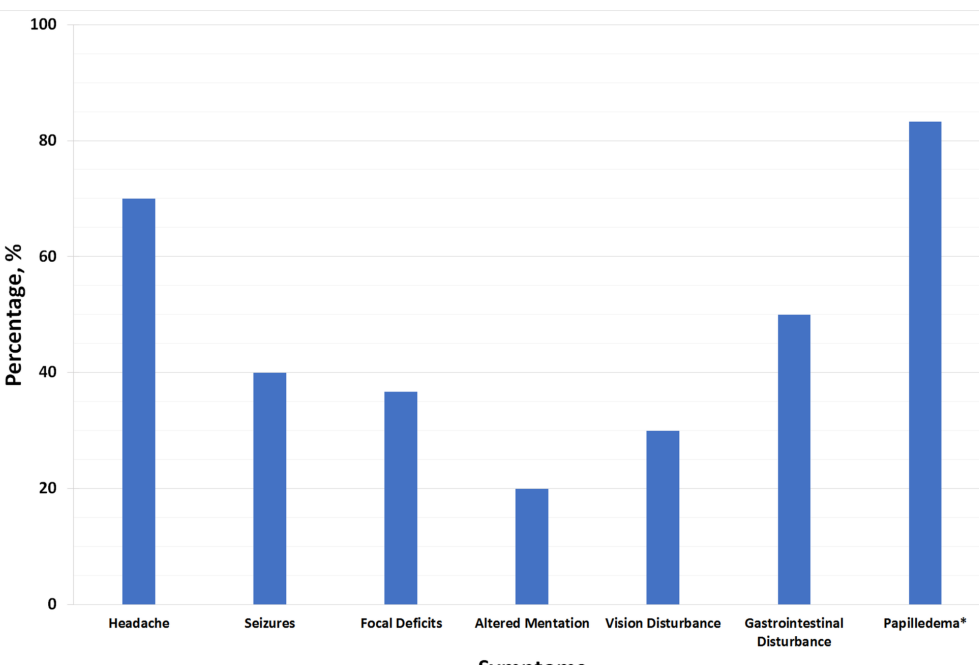

Symptoms

FIGURE 4: The presenting symptoms of cerebral venous sinus thrombosis (CVST)

${ }^{*}$ The percentage of papilledema out of 12 cases with available data

Anticoagulation abnormalities were examined in 26 cases (86.7\%). Four cases were excluded as they did not mention at least one of the following levels: antithrombin III (AT III), protein C (PC), or protein S (PS). AT III, PC, and PS were all normal in 11 cases (42.3\%). An abnormality in at least two out of the three anticoagulants was reported in six patients (23.1\%). Isolated AT III (three cases, $11.5 \%$ ), PC (one case, 3.8\%), or PS (four cases, 15.4\%) deficiency was noted in the remaining cases (Figure 5).

50

40

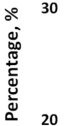
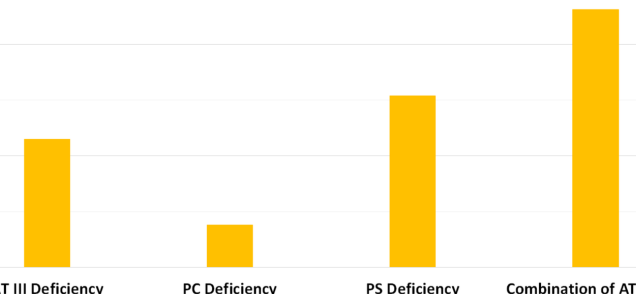

Combination of AT III, PC, Normal Levels of AT III, AT III Deficiency $\quad$ PC Deficiency

Anticoagulation Abnormality

FIGURE 5: The prevalence of different prothrombotic conditions in patients with CVST

AT III: antithrombin III; CVST: cerebral sinus venous thrombosis; PC: protein C; PS: protein S

Hyperhomocysteinemia (HHcy) was found among three (21.4\%) female patients where it was associated with a G20210A prothrombin gene mutation in the first, low PS in second, and normal AT III, PC, and PS in the third patient. Moreover, among females with HHcy, one was puerperal, the second was using EEHT, and one was puerperal and had a two-year history of EEHT use.

The G20210A prothrombin gene mutation was found among three (9.4\%) patients overall, out of which one was a male with low AT III and two were females (one with normal AT III, PC, and PS, and the other with no reported data on AT III, PC, and PS testing).

The most common initial imaging modality (22 cases, $73.3 \%$ ) and most commonly used overall (23 cases, $76.7 \%$ ) was computed tomography (CT) scan (Figure 6). Magnetic resonance venogram (MRV) was the most common modality that diagnosed or confirmed CVST (10 cases, 33.3\%). Magnetic resonance imaging (MRI) was the second most common imaging modality for initial use (five cases, 16.7\%), diagnosis or confirmation of CVST (eight cases, $26.7 \%$ ), and overall (21 cases, $70 \%$ ). 


\section{Cureus}
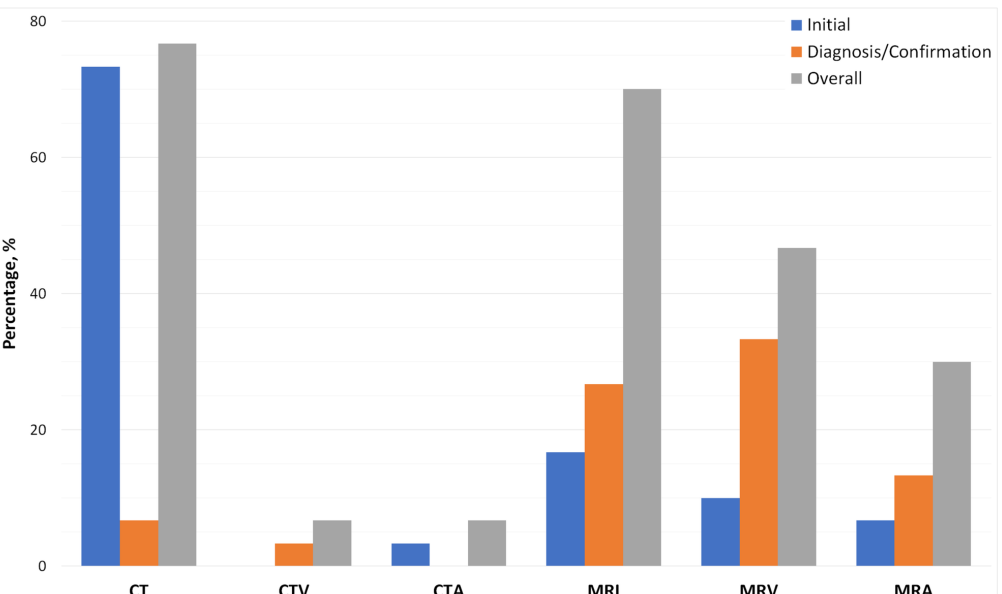

FIGURE 6: Imaging modalities used when evaluating patients with suspected CVST

Initial (blue): first imaging used to evaluate the patients. Diagnosis/Confirmation (orange): the ultimately used imaging to diagnose or confirm the diagnosis of CVST. Overall (grey): the percentage of cases where imaging used at any point in patients' evaluation

CVST: cerebral sinus venous thrombosis

Heparin agents were involved in the treatment of 18 cases (60\%), and warfarin agents were used in 10 cases (33.3\%). A heparin-warfarin combination treatment was utilized in eight cases (26.7\%). Ten cases (33.3\%) reported using other anticoagulants either with or without the use of heparin and/or warfarin agents (Figure 7). Surgical intervention occurred in three cases (10\%).

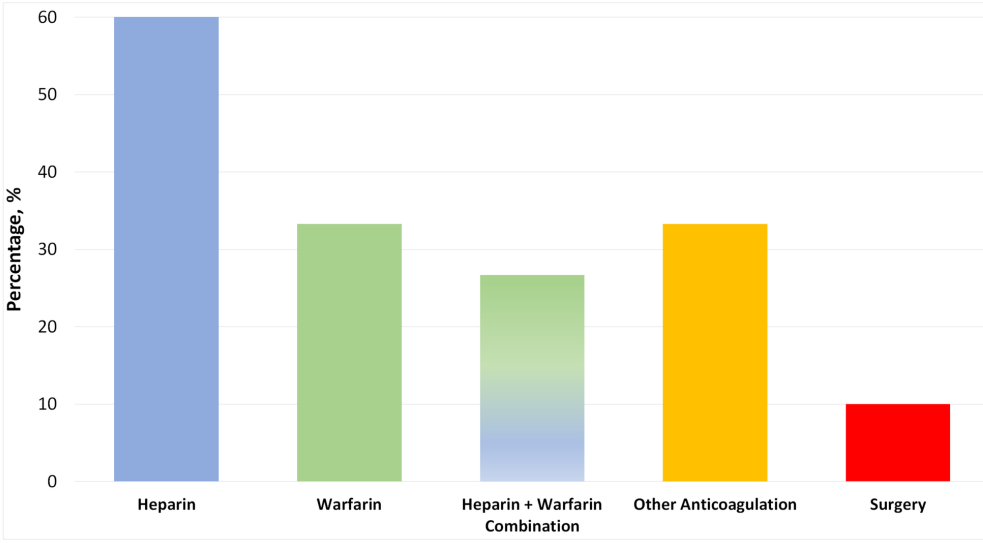

FIGURE 7: Treatments used for patients with cerebral sinus venous thrombosis (CVST)

Most patients survived (28 cases, 93.3\%), while the two remaining patients died secondary to brain death from the CVST (6.7\%). The two patients (100\%) that died were administered mannitol and corticosteroids during their treatment course, and neither were given warfarin. One other patient who survived and recovered fully was given mannitol (Table 3).

\begin{tabular}{|l|l|}
\hline Outcome & No. of patients \\
\hline Alive & $28 / 30(93.3 \%)$ \\
\hline Dead & $2 / 30(6.7 \%)$ \\
\hline
\end{tabular}

TABLE 3: Survival outcome among cerebral sinus venous thrombosis (CVST) patients

CVST occurs with similar frequency in males and females, and the symptom presentation often leads to a broad differential diagnosis. 


\section{Discussion}

In this systematic review of CVST cases, several findings are notable. CVST is a rare condition that represents a unique challenge to physicians. It occurs at a similar frequency in both men and women and has a wide variety of symptoms that are clinically indistinguishable from other common clinical conditions, which most often lead to a broad differential diagnosis [1, 32-33].

Most cases had at least one preexisting risk factor indicating multifactorial etiology with multiple mechanisms involved in its pathogenesis. Prescription drug use was the most common risk factor, including those involved in oral contraceptive use. Among females, 10 (71.4\%) reported having gender-specific risk factors. Four (40\%) were pregnant or puerperal patients and six (60\%) were getting EEHT.

Exogenous hormone therapy, pregnancy, and puerperium were the common risk factors for transient prothrombotic states and present in $78.6 \%$ of the female patients [34-36]. Tobacco use being the most common risk factor identified. More than half of the cases had symptoms between two to seven days before the presentation [37-38].

Most patients had symptoms for two to seven days at presentation and had at least two symptoms, with headache, gastrointestinal disturbance, and seizures being the most common presenting symptoms [39]. Normal AT III, PC, and PS were found in $42 \%$ of cases. Moreover, at least two of the three anticoagulants were deranged in a quarter of cases with available data [40].

The G20210A prothrombin gene mutation is linked with heightened risk for venous thrombosis, including CVST [40]. In this study, the G20210A prothrombin gene mutation was found in $9.4 \%$ of patients overall. Raised serum homocysteine levels are reported in the literature to cause a 4-fold higher risk of CVST [41]. In this study, HHcy was found among three (21.4\%) female patients with a mean age of 25 years (range: 21 30).

CT scan was the initial modality of choice for most cases and the most commonly used overall, which could be due to its easy accessibility, relatively shorter scan period, and lower cost. MRI and MRV are the second and third most commonly used imaging overall, respectively. MRI and MRV were also the two most common imaging modalities used for diagnosis or confirmation of CVST. Therefore, MRV, in combination with MRI, is a non-invasive, specific modality that has proven reliable in diagnosing CVST [42-43].

Survival rate was $93.3 \%$, and all deceased cases were not given warfarin during their treatment course. Papilledema (optic disc swelling due to high intracranial pressure) was present in $83.3 \%$ of the cases with available data. Therefore, looking for papilledema constitutes an essential factor which, unfortunately, was only reported in $40 \%$ of the cases.

\section{Conclusions}

CVST may present with a variety of clinical presentations, which makes it a diagnostic dilemma and could lead to misdiagnosis or delayed diagnosis. Appropriate physical examination by primary care providers combined with a high index of suspicion, especially in the right context, is crucial in diagnosis. We advise for increased utilization of the direct ophthalmoscope to evaluate for papilledema in patients with suspected CVST. Further well-designed studies are warranted to help determine etiologies, as well as diagnostic and management strategies, for identifying CVST cases and to establish trends in patient outcomes.

\section{Additional Information \\ Disclosures}

Conflicts of interest: In compliance with the ICMJE uniform disclosure form, all authors declare the following: Payment/services info: All authors have declared that no financial support was received from any organization for the submitted work. Financial relationships: All authors have declared that they have no financial relationships at present or within the previous three years with any organizations that might have an interest in the submitted work. Other relationships: All authors have declared that there are no other relationships or activities that could appear to have influenced the submitted work.

\section{References}

1. Guo X, Sun J, Lu X, Guan S: Intrasinus thrombolysis for cerebral venous sinus thrombosis: single-center experience. Front Neurol. 2019, 10:1185. 10.3389/fneur.2019.01185

2. Capecchi M, Abbattista M, Martinelli I: Cerebral venous sinus thrombosis. J Thromb Haemost. 2018, 16:1918-31. 10.1111/jth. 14210

3. Luo Y, Tian X, Wang X: Diagnosis and treatment of cerebral venous thrombosis: a review . Front Aging Neurosci. 2018, 10:2. 10.3389/fnagi.2018.00002

4. Heistinger M, Rumpl E, Illiasch H, Türck H, Kyrle PA, Lechner K, Pabinger I: Cerebral sinus thrombosis in a patient with hereditary protein S deficiency: case report and review of the literature. Ann Hematol.. 1992, 64:105-109. 10.1007/bf01715355

5. Musio F, Older SA, Jenkins T, Gregorie EM: Case report: cerebral venous thrombosis as a manifestation of acute ulcerative colitis. Am J Med Sci. 1993, 305:28-35. 10.1097/00000441-199301000-00006

6. Tuite P, Ahmad F, Grant I, Stewart ID, Carpenter S, Ethier R: Cerebral vein thrombosis due to hereditary antithrombin III deficiency. Can J Neurol Sci. 1993, 20:158-61. 10.1017/S0317167100047752

7. Vayá A, Larrea L, Gomis F, Mira Y, Aznar J: Cerebral venous sinus thrombosis associated with refractory anaemia with excess of blasts. Nouv Rev Fr Hematol. 1995, 37:201-203.

8. Akatsu H, Vaysburd M, Fervenza F, Peterson J, Jacobs M: Cerebral venous thrombosis in nephrotic syndrome. Clin Nephrol. 1997, 48:317-20.

9. Lefebvre P, Lierneux B, Lenaerts L, et al.: Cerebral venous thrombosis and procoagulant factors--a case study. Angiology. 1998, 49:563-71. 10.1177/000331979804900707

10. Singhal AB, Buonanno F, Rordorf G: Cerebral venous sinus thrombosis associated with hepatic cirrhosis . I Neurol Sci. 1999, 171:65-68. 10.1016/s0022-510x(99)00233-6

11. Boulos P, Kouroukis C, Blake G: Superior sagittal sinus thrombosis occurring at high altitude associated with 
protein C deficiency. Acta Haematol. 1999, 102:104-106. 10.1159/000040980

12. Akdal G, Dönmez B, Cakmakçi H, Yener GG: A case with cerebral thrombosis receiving tamoxifen treatment. Eur J Neurol. 2001, 8:723-24. 10.1046/j.1468-1331.2001.00297.x

13. Rizzato B, Ferrante F, Pisani A, Diomedi M: Recurrent cerebral venous thrombosis in a 24-year-old puerperal woman. Stroke. 2002, 33:2148-49. 10.1161/01.str.0000029380.93948.9e

14. Yilmaz S, Oren H, Irken G, Türker M, Yilmaz E, Ada E: Cerebral venous thrombosis in a patient with Evans syndrome: a rare association. Ann Hematol. 2005, 84:124-26. 10.1007/s00277-004-0963-7

15. Muthukumar N: Cerebral venous sinus thrombosis and thrombophilia presenting as pseudo-tumour syndrome following mild head injury. J Clin Neurosci. 2004, 11:924-27. 10.1016/j.jocn.2003.10.032

16. Rufa A, Cerase A, Monti L, Dotti MT, Giorgio A, Sicurelli F, Federico A: Recurrent venous thrombosis including cerebral venous sinus thrombosis in a patient taking sildenafil for erectile dysfunction. J Neurol Sci. 2007, 260:293-95. 10.1016/j.jns.2007.05.011

17. Ogata T, Kamouchi M, Kitazono T, et al.: Cerebral venous thrombosis associated with iron deficiency anemia. J Stroke Cerebrovasc Dis. 2008, 17:426-28. 10.1016/j.jstrokecerebrovasdis.2008.04.008

18. Nayak SG, Satish R, Gokulnath: Extensive cerebral venous thrombosis in a renal allograft recipient . Saudi J Kidney Dis Transpl. 2008, 19:90-93.

19. Sharpe CJ, Crowther MA, Webert KE, Donnery C: Cerebral venous thrombosis during pregnancy in the setting of type I antithrombin deficiency: case report and literature review. Transfus Med Rev. 2011, 25:6165. 10.1016/i.tmrv.2010.08.007

20. Nagesh Kumar TC, Kenchappa R, Kempegowda MB, Kulkarni A: Protein S deficiency in a case of superor sagital vein thrombosis. Indian J Med Sci. 2011, 65:36-39.

21. Skeik N, Stark MM, Tubman DE: Complicated cerebral venous sinus thrombosis with intracranial hemorrhage and mastoiditis. Vasc Endovascular Surg. 2012, 46:585-90. 10.1177/1538574412457473

22. Kolacki C, Rocco V: The combined vaginal contraceptive ring, Nuvaring, and cerebral venous sinus thrombosis: a case report and review of the literature. J Emerg Med. 2012, 42:413-16. 10.1016/j.jemermed.2011.06.011

23. Verma R, Patil TB, Kumar N: Early recanalisation of cerebral venous sinus thrombosis in an unusual case associated with severe protein S deficiency. BMJ Case Rep. 2012, 2012:bcr2012006496. 10.1136/bcr-2012006496

24. Sohoni CA, Sohoni DC: Hemicranial headache in a young adult with type 2 diabetes and migraine: a lesson learnt. Indian J Med Spec. 2014, 5:49-51. 10.7713/ijms.2013.0020

25. Lourenço Costa B, Shamasna M, Nunes J, Magalhães F, Peliz AJ: Cerebral venous thrombosis: an unexpected complication from spinal surgery. Eur Spine J. 2014, 23:253-56. 10.1007/s00586-013-3147-0

26. Giraldo EA, Arora R, Koenigsberg RA: Isolated cortical vein thrombosis associated with prothrombin gene mutation. J Stroke Cerebrovasc Dis. 2014, 23:791-93. 10.1016/j.jstrokecerebrovasdis.2013.06.004

27. Sugie M, Iizuka N, Shimizu Y, Ichikawa H: Cerebral venous thromboembolism in antiphospholipid syndrome successfully treated with the combined use of an anti-Xa inhibitor and corticosteroid. Intern Med. 2015, 54:3051-56. 10.2169/internalmedicine.54.5045

28. Gleichgerrcht E, Lim MY, Turan TN: Cerebral venous sinus thrombosis due to low-molecular-weight heparin-induced thrombocytopenia. Neurologist. 2017, 22:241-44. 10.1097/NRL.0000000000000146

29. Qadir H, Rashid A, Adil SN: Cerebral venous sinus thrombosis in a patient with undiagnosed factor VII deficiency. J Coll Physicians Surg Pak. 2017, 27:S86-88.

30. Ganeshan AS, Chango AAC: Cerebral venous sinus thrombosis: two case presentations with different clinical manifestations and multifactorial etiology. Open Sci J. 2017, 2:1-16. 10.23954/osj.v2i4.1182

31. Varner CK, Marquardt CW, Pickens PV: Antiphosphatidylserine antibody as a cause of multiple dural venous sinus thromboses and ST-elevation myocardial infarction. Am J Case Rep. 2018, 19:1042-46. 10.12659/AJCR.909698

32. Saposnik G, Barinagarrementeria F, Brown RD Jr, et al.: Diagnosis and management of cerebral venous thrombosis: a statement for healthcare professionals from the American Heart Association/American Stroke Association. Stroke. 2011, 42:1158-92, 10.1161/STR.0b013e31820a8364

33. Pabinger I, Grafenhofer H, Kyrle PA, Quehenberger P, Mannhalter C, Lechner K, Kaider A: Temporary increase in the risk for recurrence during pregnancy in women with a history of venous thromboembolism. Blood. 2002, 100:1060-62. 10.1182/blood-2002-01-0149

34. Martinelli I, Sacchi E, Landi G, Taioli E, Duca F, Mannucci PM: High risk of cerebral-vein thrombosis in carriers of a prothrombin-gene mutation and in users of oral contraceptives. N Engl J Med. 1998, 338:179397. 10.1056/NEJM199806183382502

35. Bahall M, Santlal M: Norethisterone enanthate-induced cerebral venous sinus thrombosis (CVST) . BMJ Case Rep. 2017, 2017:bcr-2017-222418. 10.1136/bcr-2017-222418

36. Shah CT, Rizqallah JJ, Oluwole O, Kalnins A, Sheagren JN: Delay in diagnosis of cerebral venous and sinus thrombosis: successful use of mechanical thrombectomy and thrombolysis. Case Rep Med. 2011, 2011:815618. 10.1155/2011/815618

37. Wasay M, Kojan S, Dai AI, Bobustuc G, Sheikh Z: Headache in cerebral venous thrombosis: incidence, pattern and location in 200 consecutive patients. J Headache Pain. 2010, 11:137-39. 10.1007/s10194-0100186-3

38. Al Hashmi K, Al Wahaibi K, Al-Khabori M, Al Lamki S: Characteristics and outcomes of patients with cerebral venous sinus thrombosis. Oman Med J. 2019, 34:434-37. 10.5001/omj.2019.79

39. Mekaj Y, Lulaj S, Daci F, et al.: Prevalence and role of antithrombin III, protein C and protein S deficiencies and activated protein $\mathrm{C}$ resistance in Kosovo women with recurrent pregnancy loss during the first trimester of pregnancy. J Hum Reprod Sci. 2015, 8:224-29. 10.4103/0974-1208.170407

40. Kim J, Martinez C, Sirotkin I: Cerebral venous thrombosis. Fed Pract. 2017, 34:33-37.

41. Martinelli I, Battaglioli T, Pedotti P, Cattaneo M, Mannucci PM: Hyperhomocysteinemia in cerebral vein thrombosis. Blood. 2003, 102:1363-66. 10.1182/blood-2003-02-0443

42. Chiewvit P, Piyapittayanan S, Poungvarin N: Cerebral venous thrombosis: diagnosis dilemma. Neurol Int. 2011, 3:e13. 10.4081/ni.2011.e13

43. Goyal G, Charan A, Singh R: Clinical presentation, neuroimaging findings, and predictors of brain parenchymal lesions in cerebral vein and dural sinus thrombosis: a retrospective study. Ann Indian Acad Neurol. 2018, 21:203-208. 10.4103/aian.AIAN_470_17 